\title{
Vortex stabilized electron beam compressed fusion grade plasma
}

\section{A. Hershcovitch}

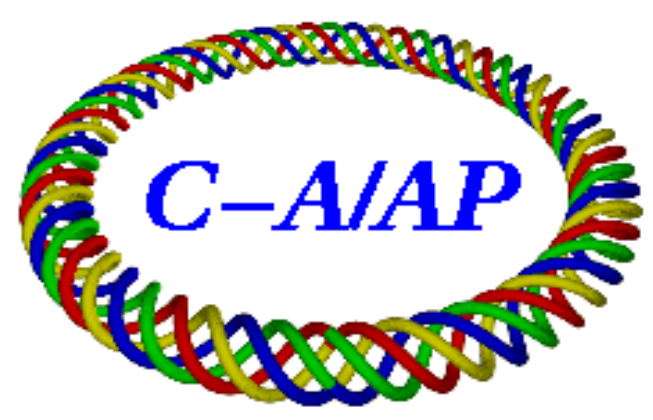

\section{Collider-Accelerator Department Brookhaven National Laboratory Upton, NY 11973}




\title{
Vortex Stabilized Electron Beam Compressed Fusion Grade Plasma
}

\author{
Ady Hershcovitch
}

Most inertial confinement fusion schemes comprise of highly compressed dense plasmas. Those schemes involve short, extremely high power, short pulses of beams (lasers, particles) applied to lower density plasmas or solid pellets.

An alternative approach could be to shot an intense electron beam through very dense, atmospheric pressure, vortex stabilized plasma. Large azimuthal magnetic generated by the electron beam is to compress and heat the plasma to fusion viable parameters. Unlike Z-pinches, this configuration should, in principle, be stable against sausage or kink instabilities; or beam - plasma instabilities (below density threshold). Based on experimental evidence beam propagation through the plasma should not be an issue.

Density of initial, pre-compression, plasma can be taken to be $5 \times 10^{23} \mathrm{~m}^{-3}$, i.e. typical plasma window density, which can easily be increased by more than one order of magnitude in the below vortex stabilized embodiment. Plasma window arc with those densities were extended into atmosphere for a few $\mathrm{cm}$ to form steady state-state a freestanding vortex stabilized arc without any enclosures [A. Hershcovitch, Phys. Plasmas 15, 057101 (2008)]. With enclosures vortex stabilized arcs can be extended for meters.

An electron beam with current $I_{z}$ generates an azimuthal magnetic field $B_{\theta}$ at a radial position $\mathrm{R}$, which is given by Ampere's law

$$
B_{\theta}=\frac{\mu_{0} I_{z}}{2 \pi R}
$$

Magnetic "pinching force" exerted on the plasma raises its pressure $P$, such that the kinetic pressure gradient is balanced by the magnetic pinching force.

$$
\nabla_{R} P=J_{Z} x B_{\theta} \text { where } \nabla_{R} P \sim \frac{P}{R} \text { and } J_{Z}=\propto \frac{B_{\theta}}{R}
$$

While $\mathrm{P}=\mathrm{nK}_{\mathrm{B}} \mathrm{T}$, where $\mathrm{n}$ and $\mathrm{T}$ are plasma density and temperature respectively; $\mathrm{K}_{\mathrm{B}}$ is the Boltzmann constant. In SI unities $\mathrm{K}_{\mathrm{B}}=1.38 \times 10^{-23}$ and $\mu_{0}=4 \pi \times 10^{-7}$.

Solving the above equations for $\mathrm{nT}$ in terms of $\mathrm{R}$,

$$
n T=4.6 \times 10^{15} \frac{I_{Z}^{2}}{R^{2}}
$$

Maximum fusion reaction rate for D-T plasma is $\langle\sigma v\rangle=1 \times 10^{-21} \mathrm{~m}^{3} / \mathrm{sec}$, where $\sigma v$ is the fusion cross section velocity product. That maximum fusion reaction rate has a broad peak for $\mathrm{T}$ between 15 to $20 \mathrm{KeV}$, or $1.74 \times 10^{8} \mathrm{~K}-2.3 \times 10^{8} \mathrm{~K}$.

Without exploring implementation details, reasonable electron beam and plasma parameters are assumed, based on which fusion power can be estimated.

Assuming a 1-meter long plasma compressed to $1 \mathrm{~cm}(0.01 \mathrm{~m})$, equation becomes,

$$
n T=4.6 \times 10^{19} I_{Z}^{2}
$$


Two electron beam currents of $1 \mathrm{MA}$ and $10 \mathrm{MA}$ are examined, for which using equation $2, \mathrm{nT}=4.6 \times 10^{31}$ and $\mathrm{nT}=4.6 \times 10^{33}$ respectively; [comparing plasma and magnetic pressures as a check (for $\mathrm{I}_{\mathrm{z}}$ of 1 and $10 \mathrm{MA}, \mathrm{B}_{\theta}=20$ and 200 Tesla respectively) equating kinetic and magnetic pressures $P=n K_{B} T=B_{\theta}^{2} / 2 \mu_{0}$, solving for $\mathrm{nT}$ also yields about the same results for $\mathrm{nT}]$.

For $\mathrm{T}=15 \mathrm{KeV}, \mathrm{n}=2.64 \times 10^{+23} \mathrm{~m}^{-3}$ and $\mathrm{n}=2.64 \times 10^{+25} \mathrm{~m}^{-3}$ respectively, in which there are, in the $1-\mathrm{m}$ long $1-\mathrm{cm}$ radius plasma are $\mathrm{N}=8.3 \times 10^{19}$ and $8.3 \times 10^{21}$ particles respectively.

Therefore, the total generated $\mathrm{D}-\mathrm{T}$ fusion power $(\mathrm{Q}=17.4 \mathrm{MeV}$ per fusion reaction) is

$P=N n<\sigma v>Q=6.1 \times 10^{10}$ Watt and $6.1 \times 10^{12}$ Watt respectively (for $1 \mathrm{MA}$ and $10 \mathrm{MA}$ beams).

With readily available electron beam technology, net power cannot be generated by this method, since electron beam energy will need to be in the multi-MeV range, i.e. beam power will exceed fusion power by more than an order of magnitude.

But, since the plasma window was shown to separate 9 atmosphere gas targets from vacuum [A. Hershcovitch, Physics of Plasmas, 5, 2130 (1998)], it is not inconceivable to start with a pre-compression pressure of 9 atmosphere, i.e. with a density of $4.5 \times 10^{24} \mathrm{~m}^{-3}$. For the same compression factor with a $10 \mathrm{MA}$ electron beam, close to breakeven conditions can be reached. Higher electron beam currents are reached at relatively lower extraction voltages, since electron beam current scale as $\mathrm{V}^{3 / 2}$ (non-relativistic). Higher pre-compression densities are also possible. However, when considering the efficiencies of thermal to electrical conversion of fusion energy and generating the electron beam, as well as the energy required to produce the target plasma, the scheme viability as an electrical power generating reactor does not seem to be promising.

Nevertheless there is a glimmer of hope with a plasma cathode electron beam that was developed a while ago (for which DOE has a patent: A. Hershcovitch, V.J. Kovarik, K. Prelec, U.S. Patent 4,942,339, July 17, 1990, Intense High Brightness Steady State Electron Beam Generator), since with plasma cathode electron gun (nonrelativistic) a perveance of over 280 microperv was achieved (factor of about 300 larger than high perveance guns), and since there are relativistic enhancements to the Child law, MA electron beams could be extracted at below MV. More details regarding that electron gun can be found at A. Hershcovitch, "Extraction of superthermal electrons in a high current, low emittance, steady state electron gun with a plasma cathode," Appl. Phys. Lett. 68, 464 (1996). An electron beam can then be focused into a plasma target, where any space charge limitations are neutralized.

However, further R\&D is required for such electron beam technology development. 


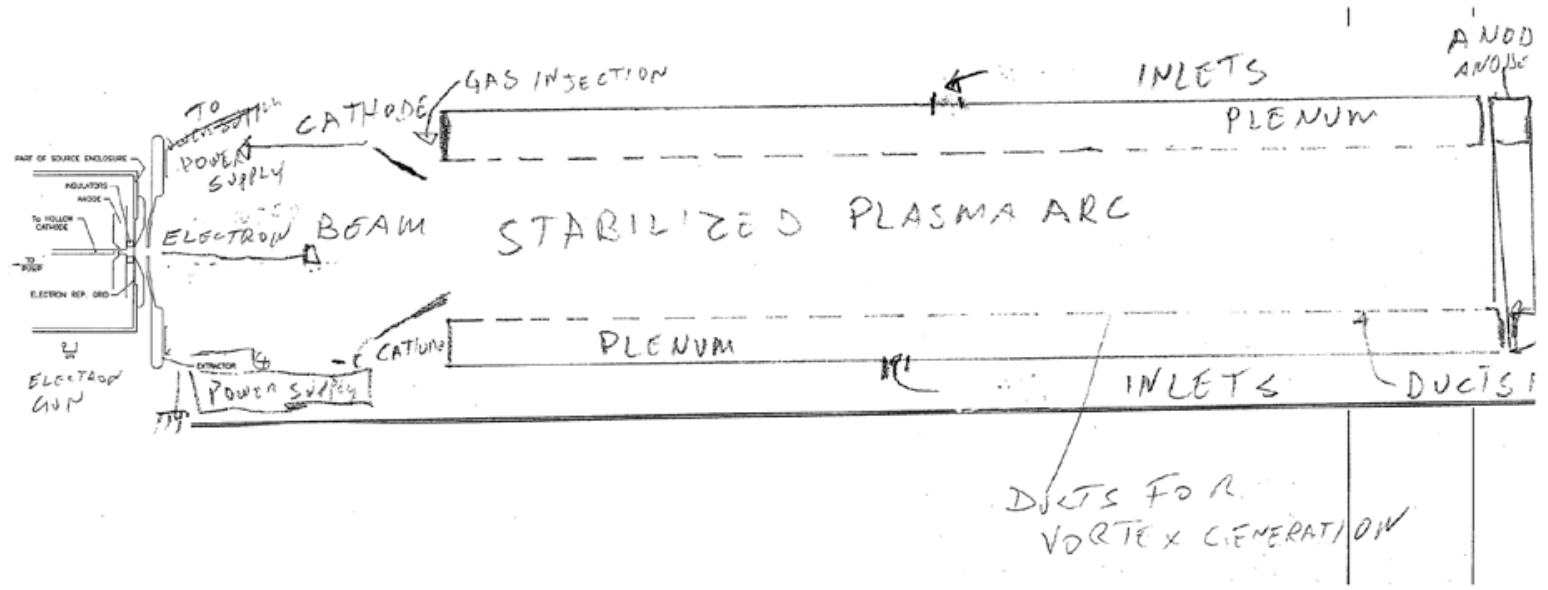

Possible embodiment of the fusion grade plasma generation concept; plasma cathode electron gun is displayed in the above figure. 